\title{
LOCAL OCULAR COMPENSATION FOR IMPOSED LOCAL REFRACTIVE ERROR
}

\author{
F. A. Miles and J. Wallman \\ Department of Biology, City College of CUNY, 138th Street and Convent Avenue, \\ New York, NY 10031, U.S.A.
}

(Received 21 March 1989; in revised form 10 July 1989)

\begin{abstract}
Chicks were raised in a low-ceiling environment to find out if their eye growth could compensate for locally imposed hyperopic refractive errors. These chicks became selectively more myopic in the upper visual field than chicks raised in a high-ceiling environment. The vitreous chamber in the low-ceiling birds showed a selective elongation in the ventral region that was not seen in the eyes of the high-ceiling birds. This morphological difference was small, but probably adequate to account for the additional myopia in the low-ceiling birds. These results are consistent with the idea of a visually mediated growth mechanism regulating the local refractive state across the entire visual field so that it matches the customary viewing conditions. Such a mechanism might account for the finding of Fitzke, Hayes, Hodos, Holden and Low (Journal of Physiology, London, 369, 33-44, 1985) that the refractive errors in the lower field are exactly appropriate for focusing the image of the ground on the retina.
\end{abstract}

Emmetropization Myopia Birds Eye growth "Ramp retina"

\section{INTRODUCTION}

At hatching, the chick's eyes are normally slightly hyperopic when refracted along the optic axis and, over a period of weeks, gradually become emmetropic (Wallman, Adams \& Trachtman, 1981; Wallman \& Adams, 1987). Deprivation of form vision during this developmental period results in severe myopia, mainly due to axial elongation of the vitreous chamber (Wallman, Turkel \& Trachtman, 1978; Yinon, Rose \& Shapiro, 1980; Hodos \& Kuenzel, 1984; Hodos, Fitzke, Hayes \& Holden, 1985; Hayes, Fitzke, Hodos \& Holden, 1986; Osol, Schwartz \& Foss, 1986; Lauber \& Oishi, 1987; Troilo, Gottlieb \& Wallman, 1987; Wallman, Gottlieb, Rajaram \& Fugate-Wentzek, 1987; PickettSeltner, Weerheim, Sivak \& Pasternak, 1987; Gottlieb, Fugate-Wentzek \& Wallman, 1987; Schaeffel, Glasser \& Howland, 1988; PickettSeltner, Sivak \& Pasternak, 1988). Other animals, including humans, also show myopia and ocular enlargement when deprived of form vision during development (Robb, 1977; Sherman, Norton \& Casagrande, 1977; Wiesel \& Raviola, 1977, 1979; Wilson \& Sherman, 1977; Raviola \& Wiesel, 1978; McKanna \& Casagrande, 1978; Sommers, Kaiser-Kupfer \& Kupfer. 1978; O'Leary \& Millodot, 1979; Hoyt, Stone, Fromer \& Bildon, 1981; Rabin, van Sluyters \& Malach,
1981; Johnson, Post, Chalupa \& Lee, 1982; Kirby, Sutton \& Weiss, 1982; Nathan, Kiely, Crewther \& Crewther, 1985; Smith, Harwerth, Crawford \& von Noorden, 1987).

One suggestion is that deprivation of form vision disables a growth control mechanism that is visually mediated and normally functions to match the size of the eye to its optical power so that the image plane coincides with the retina. Schaeffel et al. (1988) recently provided evidence for such a mechanism by showing that chicks raised with lenses in front of their eyes can compensate appropriately: eyes made functionally myopic by positive lenses tended to grow towards hyperopia and eyes made functionally hyperopic by negative lenses tended to grow towards myopia. Further, this compensation still occurred in animals that lacked the ability to accommodate following lesions of the Edinger-Westphal nucleus (Schaeffel, Troilo, Wallman \& Howland, paper under review). That the growth control mechanism can successfully compensate for positive or negative refractive errors is also apparent in the experiments of Troilo (1989), who found that chicks could recover from both the myopia associated with form deprivation and the hyperopia associated with dark rearing. Remarkably, this recovery could still be initiated even after 
sectioning of the optic nerve (though recovery here was never complete and showed overshooting).

A striking characteristic of this growth control mechanism in birds is that it can operate locally. Working with chicks, Hodos and Kuenzel (1984) reported that eyes totally deprived of pattern were significantly larger than controls in both the axial and equatorial dimensions, while eyes so deprived only in the frontal field were significantly enlarged only in the equatorial dimension. These authors suggested that local deprivation might cause local myopia, and subsequent studies have amply confirmed this idea: depriving only a portion of the retina of patterned input results in myopia and excessive growth only in the deprived region (Wallman \& Adams, 1987; Wallman et al., 1987; Gottlieb et al., 1987). In addition, local myopia and ocular enlargement could be induced by local form deprevation even after optic nerve section (Troilo et al., 1987; Wildsoet \& Pettigrew, 1988).

One function of this local growth control mechanism in birds might be to sculpt the eye so that the retina is conjugate with the ground (Wallman et al., 1987; Wildsoet \& Pettigrew, 1988). For an observer standing on a flat plain, the viewing distance to any point on the ground, $d$, is given by the ratio of the height of the observer's eye above the ground, $H$, and the sine of the angle of elevation below the horizon, $\theta$, i.e. $d=H /(\sin \theta)$. Thus, for an emmetropic eye with accommodation relaxed, any given point on the ground would be out of focus in the hyperopic direction by the amount, $(\sin \theta) / H$. This blur could be cleared either locally by accommodation or globally by the eye being progressively more myopic in the lower field. Fitzke et al. (1985) found that the pigeon's eye was generally emmetropic at and above the horizontal meridian but myopic in the lower visual field. Further, this myopia increased as the sine of the angle of eccentricity and a linear regression of refractive error on $\sin \theta$ had a slope approximating the height of the bird's eye above the ground (assuming that the bird stands erect). This indicates that if a pigeon were standing on a flat plain with relaxed accommodation, the retinal image of the ground extending from immediately beneath the animal to the distant horizon would all be in focus simultaneously. Similar refractive anisotropies have been found in other species (Walls, 1942; Sivak, 1976; Erichsen \& Hodos, 1989).
The clear suggestion above is that the process mediating emmetropization in birds us under local control. If this is the case, then one would expect that the growth of the bird's eye would be sensitive to a disruption in the threedimensional layout of its visual world. To test this possibility we placed chicks in an environment that rendered the ventral retina hyperopic and sought evidence for selective compensatory growth towards myopia in this retinal region. Various studies have suggested that animals raised in confined spaces are more myopic than usual, though the proximity of the surroundings is only one of several factors that might have been different from normal in these environments (Young, 1961a, 1963; Rose, Yinon \& Belkin, 1974). We here report that chickens that have been raised from hatching in a low-ceiling environment tend to be more myopic in the upper field than chickens raised in a highceiling environment. Further, this local myopia is associated with local enlargement of the vitreous chamber.

\section{METHODS}

\section{Subjects and treatments}

White Leghorn chicks (Gallus gallus domesticus) were hatched in the laboratory (eggs obtained from Shamrock Poultry Farms, N.J.) and raised in temperature controlled enclosures on a $14: 10 \mathrm{hr}$ light:dark cycle for periods up to 12 weeks. Food and water were always freely available. Two enclosures were used, each $1.2 \times 1.2 \mathrm{~m}$ across and $0.9 \mathrm{~m}$ high with mirrored walls to extend the visual horizon. In one case, which we refer to as the high-ceiling environment, there was no top to the enclosure so that the ceiling of the room, which was covered with densely patterned and coloured cloth, was the nearest overhead visual feature. The ceiling was approx. $3 \mathrm{~m}$ above the floor. (At about 8 weeks, it was necessary to extend the walls of this enclosure upwards $0.6 \mathrm{~m}$ with wire mesh to prevent the birds from escaping.) The other enclosure, which provided a low-ceiling environment, had an adjustable ceiling made of patterned cloth stretched across a wooden frame; this frame was suspended by a pulley system which was adjusted from time to time to keep the ceiling approx. $5 \mathrm{~cm}$ above the heads of the growing birds (when they stood erect). An incandescent lamp transilluminated the coloured, patterned cloth from above so that the 
luminance levels within the enclosure were comparable with those in the high-ceiling enclosure. The air inside both enclosures was replenished by a fan with a thermostatically controlled heater.

Two experimental protocols were followed. In the first, 12 birds were raised in the lowceiling environment for 8 weeks (experimental animals) and then 12 more were raised in the high-ceiling enclosure for a similar period of time (controls). Refractive errors were measured at the conclusion of each experiment. A potential problem with the data from this initial investigation is observer bias since the two groups of animals were raised at different times and the refractionist was aware of the differences in the rearing conditions. To preclude this possibility, a second investigation was carried out following a protocol that involved masked measurements: the two groups of birds were raised concurrently and refracted at 8 and 12 weeks of age, the refractionist not knowing from which enclosure any given animal had been taken. The refraction data obtained with these two protocols were in agreement on all essentials and only those obtained with the blind procedure will be presented in full. The data obtained in the first study will only be presented in summary form and will be clearly identified.

\section{Measurement of refractive error}

Chicks were anesthetized with an intramuscular injection of a mixture of $3.5 \%$ chloral hydrate, $3 \%$ sodium pentobarbital, $5 \%$ propylene glycol, and $2.5 \%$ ethanol in distilled water $(0.33 \mathrm{ml} / 100 \mathrm{~g}$ body $\mathrm{wt})$. Cycloplegia was produced with a solution of curare, atropine sulphate, benzalkonium chloride and polyvinyl alcohol, topically applied to the cornea (Wallman \& Adams, 1987). Generally, 10-15 drops, one drop/5 $\mathrm{min}$, were required before pupillary responses to a bright light were eliminated. The bird was then placed in a stereotaxic instrument mounted on a turntable that allowed rotation of the animal about a horizontal axis passing through the right eye nearly orthogonal to its optic axis. As vertical rotation about the optic axis causes the measuring beam to fall on the pecten, we used an azimuthal angle slightly posterior to the optic axis: $70^{\circ}$ lateral to the rostro-caudal axis of the head. The head was oriented so that the upper beak rested on a bar positioned at the anterior margin of the external nares and $1 \mathrm{~mm}$ below the ear bars. After measuring the refractive error of the right $0^{\circ}, 15^{\circ}, 30^{\circ}, 45^{\circ}$ and $60^{\circ}$, above and below the stereotaxic horixontal, the animal was rotated $180^{\circ}$ from the prone to the supine position, and the same measures carried out on the left eye. Four measurements were made at each angle of elevation.

Refractive error was measured with two different devices. The first measures at 8 weeks were done with a Hartinger Coincidence Refractometer (Jena), equipped with an accessory lens to permit the refraction of small eyes as previously described (Wallman \& Adams, 1987). This device directs two pencils of light, separated by approx. $2 \mathrm{~mm}$, into the pupil and hence samples only the optics along these discrete light paths. During off-axis refractions above and below the horizontal, the eccentrically viewed pupil showed considerable vertical narrowing so that the refractometer had to be oriented with the two pencils in the same horizontal plane. Thus, eyes could only be refracted in the horizontal plane, and it was not possible to gain any estimate of astigmatism.

To obtain a more complete sampling of the overall optics, the 12 week measures of refractive error were performed with a purpose-built device that was an extensively modified version of the photoretinoscope of Schaeffel, Farkas and Howland (1986). With this method, the bird's eye was illuminated by two vertical rows of lights, one red and one green, arranged side by side, $12 \mathrm{~mm}$ apart. Each row was created by a fiber optic array and was approx. $2 \mathrm{~mm}$ wide and $20 \mathrm{~mm}$ long. When the bird's eye was viewed from between these two rows of lights, the reflected retinal image appeared red on one side and green on the other, depending on whether the reflecting layer of the bird's retina was brought to a focus in front of or behind the plane of the observer. By interposing lenses as in standard retinoscopy it was possible to bring the plane of focus into the plane of the observer's eye, allowing the refractive state to be estimated. At this "color-reversal" point, the clear partitioning into red and green zones disappears. The two rows of lights were at a distance of $74.5 \mathrm{~cm}$ to the bird's eye and, to help the observer see the color transitions, the retinal reflex was viewed through a telescope situated between the lights. Front surface mirrors were used to fold the light path, allowing the observer to hold the test lenses immediately in front of the bird's eye. Using this technique, it was generally possible to resolve the "colorreversal" point to $0.5 \mathrm{D}$. However, the time 
required to determine each reversal point (up to $5 \mathrm{~min}$ ) did not allow more than one measure at each angle of eccentricity before corneal drying began to obscure the reflex. A further limitation of this method was that the partitioning of the $\mathrm{red} / \mathrm{green}$ zones was not always clear, especially at the most extreme eccentricities $\left(60^{\circ}\right)$, so that complete data sets could not be obtained for all eyes.

Both of the refractometers used in this study effectively locate the plane of focus of the reflecting layer of the retina, which lies in front of the photoreceptors and therefore introduces a systematic error (Glickstein \& Millodot, 1970; Hughes, 1979). No attempt was made to correct for these hyperopic errors.

\section{Measurement of eye shape}

After the final refractions, chicks were anesthetized, perfused with $5 \%$ buffered formalin, and their heads stored in 5\% formalin. The heads were then placed back in the stereotaxic instrument and the dorsal extremity of each eye marked to permit accurate orientation of the eye after its subsequent enucleation. After excising the eyes and removing all adherent extraocular tissue, each eye was photographed from the temporal aspect to define the dorso-ventral outline. The photographs were projected onto a digitizing tablet and the outline of each eye traced by hand. The outlines from each group were then aligned and averaged using previously described methods (Gottlieb et al., 1987; Troilo \& Wallman, 1987; Wallman et al., 1987).

We wished to estimate the depth of the vitreous chamber at different angles of eccentricity and to compare these measurements with the corresponding refractive errors. Unfortunately, in a compound optical system such as the eye, the distance to the retina that corresponds to a particular off-axis refraction cannot be determined by simply drawing a line at the angle of refraction: it is necessary to measure the distance to the retina from the second principal point of the optical system, while measuring the angle from the second nodal point, in order to account for the magnification of angles by the eye's optical system. The second principal point is approximately located at the mid-point of the line joining the dorsal and ventral margins of the cornea (Wallman \& Adams, 1987). As shown in Fig. 3A, the perpendicular bisecting this line defines the "axis of symmetry" and, based on the estimate of Schaeffel and Howland (1988), the second nodal point was assumed
A HIGH CEILING

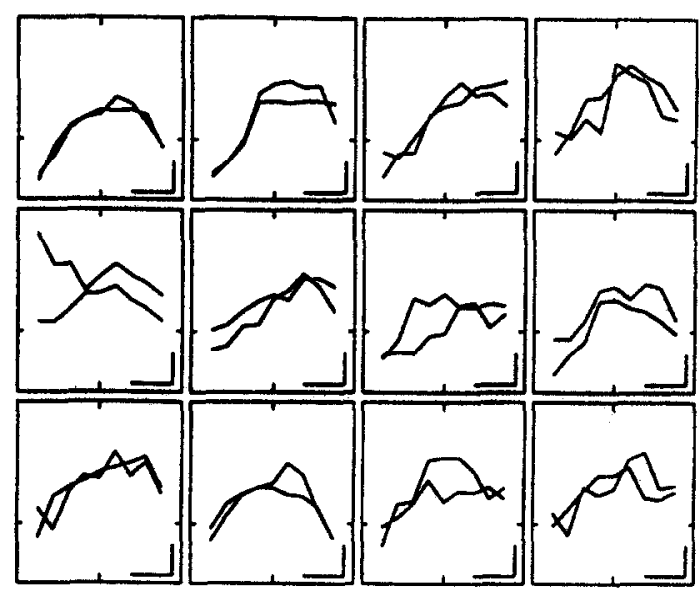

B LOW CEILING

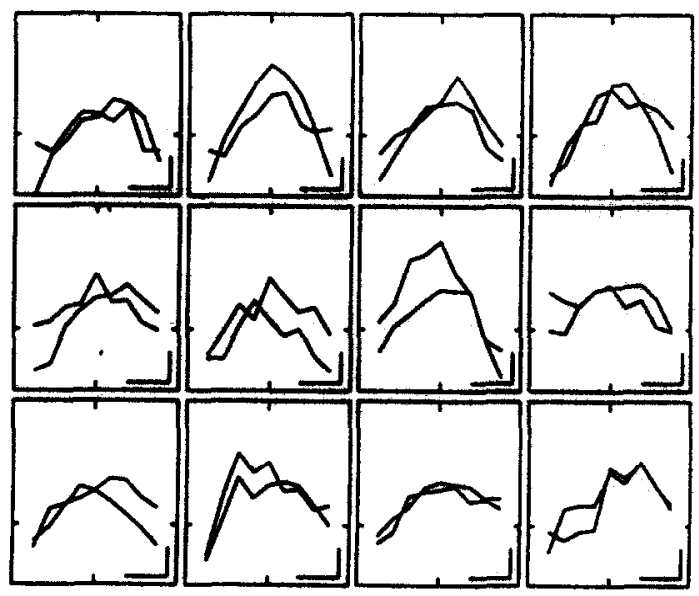

Fig. 1. Dependence of refractive errors (ordinate) on angle of elevation (abscissa): data from individual eyes measured at 8 weeks of age with a Hartinger Refractometer. (A) High-ceiling birds. (B) Low-ceiling birds. Each box contains data for one bird (2 eyes). Ticks indicate zero refractive error and zero angle of elevation. Positive angles represent the upper visual field. The refractive errors have not been corrected for the artifect of retinoscopy. Calibration bars are $2 \mathrm{D}$ and $40^{\circ}$.

to be located on this axis at a distance from the sclera that was $60 \%$ of the axial length $(+0.4 \mathrm{~mm})$. This measure is probably accurate only close to the optic axis; consequently morphological measurements can be expected to correlate well with refractions only at small angles of eccentricity (perhaps $<30^{\circ}$ ). Even the most recent attempt to model the optics of the chicken's eye failed to account for peripheral refractions (Schaefiel \& Howland, 1988). Thus, it is not possible at present to make quantitative predictions about the amount of ametropia at the more eccentric locations. 
RESULTS

\section{Refractive errors}

The dependence of refractive error on the angle of elevation for individual eyes at 8 weeks of age is shown in Fig. 1. Although these plots show considerable individual variability, it is clear that refractive errors tended to be progressively more myopic with increasing eccentricity, especially in the lower visual field. The tendency towards myopia in the upper visual field was more pronounced in the low-ceiling birds, while that in the lower visual field was similar in both groups. For 19 of 24 eyes from the high-ceiling birds, upper field refractions were similar to those on the horizon, whereas for 20 of 24 eyes from the low-ceiling birds, upper field refractions were roughly symmetric with those in the lower field.

Lower visual field. Eyes were progressively more myopic with increasing eccentricity in the lower visual field regardless of the height of the ceiling (Fig. 2). Student's $t$-tests on the slopes of the regression lines for refractive error vs angle of evaluation in the lower visual field failed to reveal any significant differences between the birds raised in the high- and low-ceiling environments. Further, an analysis of variance also failed to reveal any effect of the environment on refractive errors in the lower visual field. Specifically, an ANOVA with repeated measures
(Dixon, 1983) on refractive error in the lower visual field at 8 weeks of age showed no significant interaction between angle of elevation and ceiling height, $F(4,88)=1.29, P>0.05$ (Huynh Feldt), with a significant main effect of angle of elevation, $F(4,88)=128.93, \quad P<0.0001$ (Huynh Feldt), but no significant main effect of ceiling height, $F(1,22)=0.26, P>0.05$. Because if missing data points (see Methods), the ANOVA on refractive error at 12 weeks of age was only possible for a subset of animals: 8 from the high-ceiling group and 7 from the low-ceiling group. Nonetheless, the ANOVA on this restricted sample led to essentially the same conclusion, with no significant interaction between angle of elevation and ceiling height, $F(4,52)=0.65, P>0.05$ (Huynh Feldt), a significant main effect of angle of elevation, $F(4,52)=73.31, P<0.0001$ (Huynh Feldt), but no significant main effect of ceiling height, $F(1,13)=1.10, P>0.05$.

The dependence of refractive error on angle of elevation in the lower visual field was less pronounced at 12 weeks of age than at 8 weeks, regardless of the environmental conditions. Thus, for the high-ceiling birds, mean rates of change of refractive error with angle of elevation in the lower visual field, based on linear regression, were 0.062 and $0.041 \mathrm{D} / \mathrm{deg}$ at 8 and 12 weeks of age, respectively, and these differences were statistically significant $(P=0.0087$,
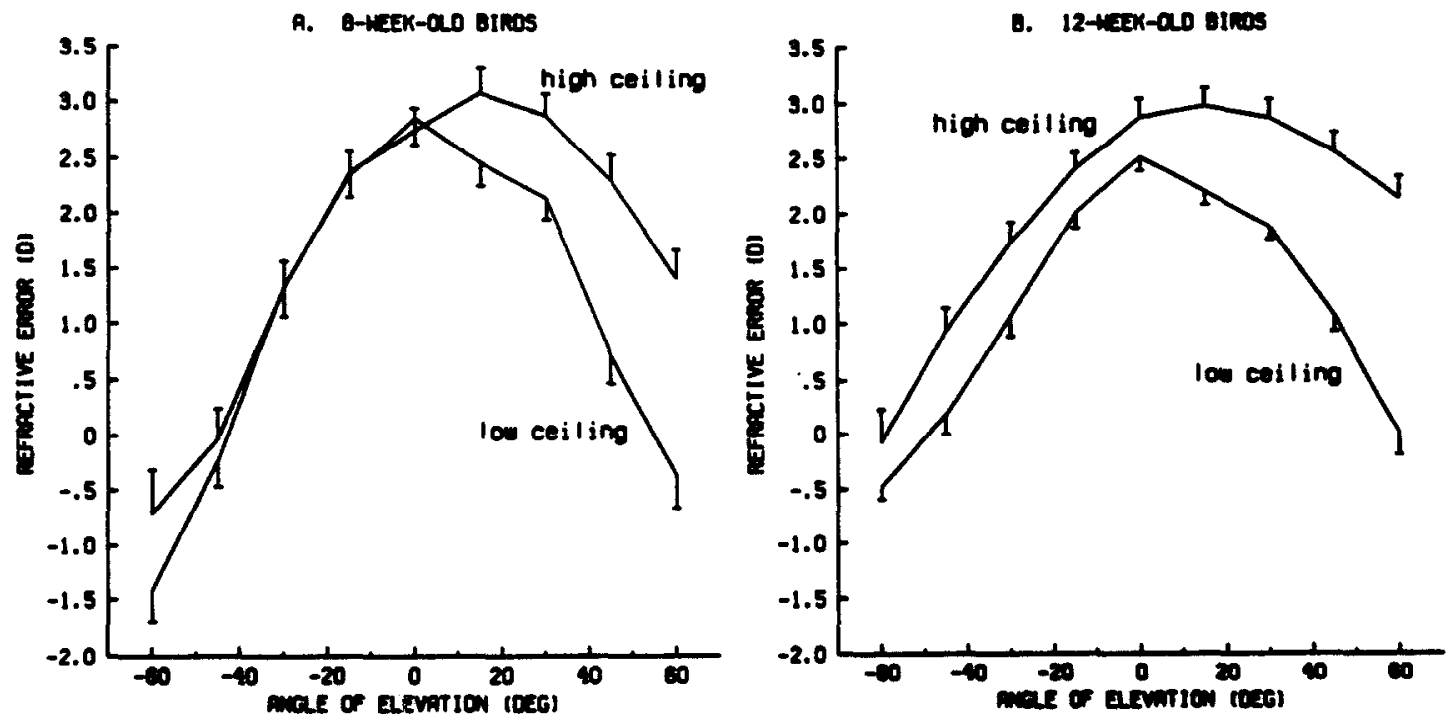

Fig. 2. Dependence of mean refractive error on angle of elevation. (A) Eight week old birds (Hartinger Refractometer). (B) Twelve week old birds (modified Photoretinoscope). Positive angles reprexent upper visual field. Error bars (SEM) are shown upward for the high-ceiling birds and downward for the low-ceiling birds. Notice that the upper and lower field refractions become progressively further apart in the high-ceiling animals, but not so in the low. 
$t$-test). The situation was very similar in the birds raised with the low ceiling, the corresponding regression coefficients being 0.074 and $0.052 \mathrm{D} / \mathrm{deg}$, and once more the differences were highly significant $(P=0.0014, t$-test). It has been suggested that, in pigeons, the refractive errors in the lower visual field depend on the height of the animal's eye above the ground (Fitzke et al., 1985), and this might account for the age-dependence of the slopes in our experiments since our birds were clearly taller at 12 weeks than at 8 weeks. If this is the explanation, then the refractive error should be a linear function of the sine of the angle of elevation $(\sin \theta)$, and the slope of the relationship should approximate the height of the eye above the ground (see Introduction). In fact, linear regression of mean refractive error on $\sin \theta$ below the horizontal meridian revealed mean slopes of $19 \mathrm{~cm}(r=1.00)$ at 8 weeks of age and $24 \mathrm{~cm}$ $(r=0.98)$ at 12 weeks for the high-ceiling birds. Corresponding slopes for the low-ceiling birds were $16 \mathrm{~cm}(r=0.99)$ at 8 weeks of age and $24 \mathrm{~cm}(r=1.00)$ at 12 weeks. We did not measure the height of the bird's eyes above the ground directly but we can obtain a crude estimate for the low-ceiling birds because, as stated in the Methods, we attempted to keep the ceiling approx. $5 \mathrm{~cm}$ above the upright birds' heads and took note of the required setting. At the time that the 8 and 12 weeks measures were made the ceilings were 30 and $35 \mathrm{~cm}$, respectively, above the floor. This suggests that the eye heights of these birds were $<25$ and $<30 \mathrm{~cm}$, respectively, which encompasses the estimate obtained from the refraction data.

Upper visual field. It is clear from Fig. 1 and 2 that the chickens' eyes also tended to be more myopic in the upper visual field than along the horizontal meridian, an effect previously observed in the pigeon by Erichsen (1979). Of particular interest here is the fact that this myopic trend in the upper visual field was more prominent in the birds raised in the low-ceiling environment. For example, at $60^{\circ}$ eccentricity, low-ceiling birds were on average more myopic than high-ceiling birds by $1.8 \mathrm{D}$ at 8 weeks of age and by $2.1 \mathrm{D}$ at 12 weeks of age. Further, an ANOVA on refractive error in the upper visual field at 8 weeks of age showed a significant interaction between angle of elevation and ceiling height, $F(4,88)=8.38, P=0.0004$ (Huynh Feldt). Similar results were obtained at 12 weeks of age-on the smaller data set mentioned above - with a significant interaction between angle of elevation and ceiling height, $F(4,80)=11.48, \quad P=0.0002$ (Huynh Feldt). Thus, the tendency for refractive errors in the upper visual field to be more myopic in the low-ceiling birds than in the high-ceiling birds was statistically highly significant.

All of the foregoing data were obtained using a protocol in which the refractionist worked "blind" (see Methods). However, almost identical results were obtained in a prior experiment using the same procedures except that the refractionist was aware of the environmental conditions that each bird had experienced (see Methods). An ANOVA on the refractive error in the upper visual field at 8 weeks of age in this earlier study showed a significant interaction between angle of elevation and ceiling height, $F(4,76)=34.02, P=0.01$ (Huynh Feldt). At $60^{\circ}$ eccentricity, for example, the low-ceiling birds were on average $2.1 \mathrm{D}$ more myopic than the high-ceiling birds, a difference which was very similar to that seen in the 8 week birds in the later study. Thus, the two studies agreed in all essentials: eyes rendered in effect hyperopic in the upper visual field by the low ceiling became selectively more myopic in that region when compared to the eyes of control animals raised with the high ceiling.

\section{Eye shapes}

Consistent with the relative myopia in the upper field of the low-ceiling birds, we found a relative elongation of the ventral part of the vitreous chamber in these same animals. The analysis of this change in the shape of the eye is complicated by the fact that the birds raised with the low ceiling were significantly smaller than those raised with the high ceiling. This is apparent from their body weights, which at 12 weeks of age ranged from 0.84 to $1.33 \mathrm{~kg}$ (mean, $1.02 \mathrm{~kg}$ ) in the low-ceiling group, and from 0.92 to $1.44 \mathrm{~kg}$ (mean, $1.28 \mathrm{~kg}$ ) in the highceiling group, and the differences were statistically significant $(P<0.0002$, $t$-test $)$. The lowceiling birds also had slightly smaller eyes and this is evident from the profile drawings in Fig. 3(A) and from the estimated depths of the vitreous chambers at each angle of eccentricity plotted in Fig. 3(B). We have no explanation for this large difference in the growth of the two groups. However, our major concern was with any selective effect of the ceiling height on eye 
growth and, in particular, with the possibility that the increased myopia in the upper visual field of the low-ceiling birds might be due selective enlargement of the ventral portion of the vitreous chamber. It is clear from the vitreous chamber depth profiles in Fig. 3(B) that the eyes of the low-ceiling birds were not simply scaled-down versions of the eyes of the highceiling birds but showed different dorso-ventral asymmetries. In fact, the eyes of the low-ceiling birds show a bulge in the ventral hemiretina that has no equivalent in the eyes of the high-ceiling birds.

To examine these asymmetries quantitatively it was necessary to factor out the size differences between the high- and low-ceiling groups. This was achieved by estimating the difference between the depths of the vitreous chamber in the dorsal and ventral halves of the eyes. Accordingly, for each eye the depth of the vitreous chamber at each angle of eccentricity in the ventral retina was subtracted from the depth at

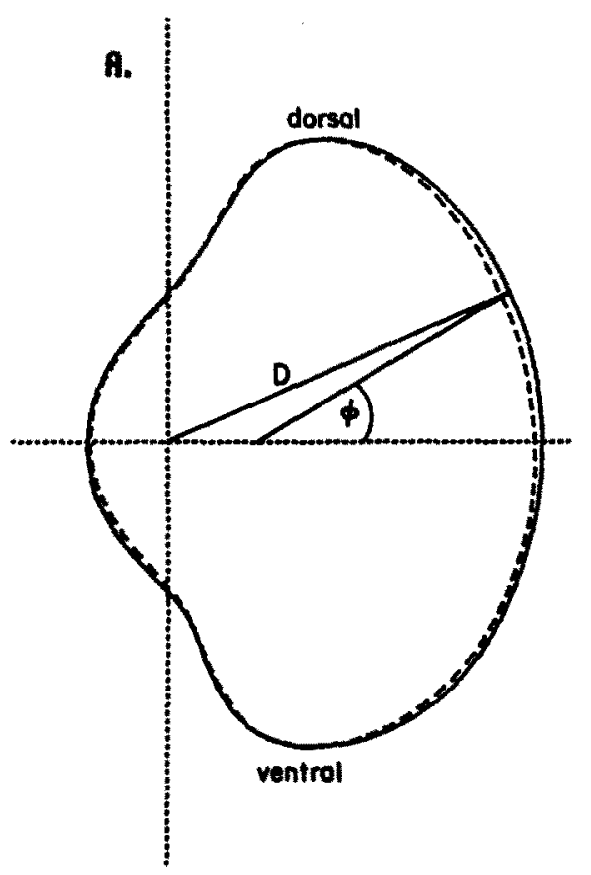

B.

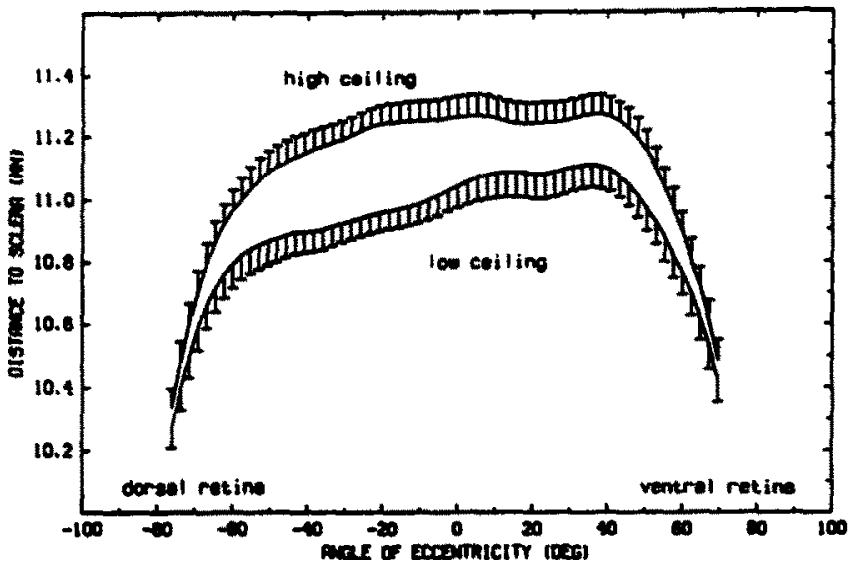

c.

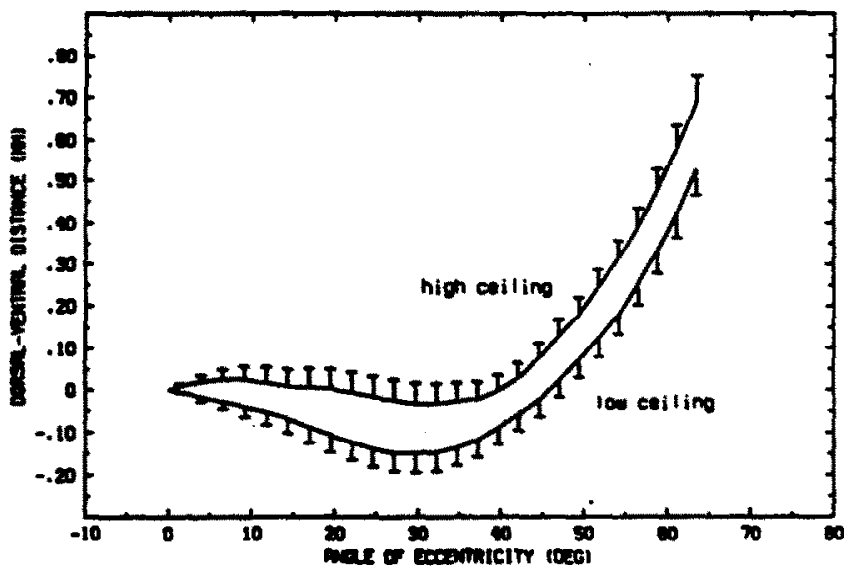

Fig. 3. Dorso-ventral eye shapes. (A) Mean profiles traced from photographs and aligned so that dorsal retina is uppermost; data for high-ceiling birds $(n=11)$ shown in continuous line and data for low-ceiling birds $(n=10)$ in dashed line. $\phi$, angle of eccentricity $\left(30^{\circ}\right)$ from estimated posterior nodal point; $D$. distance to the sclera. (B) Dependence of depth of vitreous chamber (as indicated by mean distance to sclera) on angle of eccentricity; positive angles indicate dorsal retina. Note that the angles are measured from the second nodal point of the eye, and probably approximate the angles of the refractions (Figs 1 and 2) up to 30: but deviate from them increasingly as the eccentricity increases beyond this value (see text). Error bars (SEM) are shown upward for the high-ceiling birds and downward for the low-ceiling birds. (C) Dorso-ventral asymmetry: the distance to the sclera at each angle of eccentricity in the dorsal retina was subtracted from the distance to the selera at the corresponding angle in the ventral retina and the means of these differences plotted against the angle of eccentricity. Error bars (SEM) are shown upward for the high-ceiling birds and downward for the low-ceiling birds. 
the corresponding angle in the dorsal retina.* Means and standard errors were then calculated and are shown in Fig. 3(C). It is apparent from this figure that the eyes of the high-ceiling birds showed significant dorso-ventral asymmetry only at eccentricities $>40^{\circ}$, as the dorsal measures gradually exceeded the ventral ones by increasing amounts. This is only roughly in accord with the refractive errors in these birds, which were always more myopic in the lower visual field than in the 'upper and by amounts that increased steadily with increasing eccentricity. The measures of dorso-ventral asymmetry for the eyes of the low-ceiling birds show a similar pattern though the entire curve is slightly lower, indicating that the eyes of the low-ceiling birds had a ventral protuberance that was not present in the eyes of the highceiling birds. Furthermore, this protuberance was statistically significant: an ANOVA on the dorsal-ventral differences in the depth of the vitreous chamber showed no significant interaction between angle of eccentricity and ceiling height, $F(26,1040)=0.71, P>0.05$ (Huynh Feldt), but a main effect of angle of elevation, $F(26,1040)=111.86, P<0.0001$ (Huynh Feldt) and of ceiling height, $F(1,40)=5.51, P=0.024$ (Huynh Feldt).

\section{DISCUSSION}

Chicks raised in the low-ceiling environment were selectively more myopic in the upper visual field than chicks raised in the high-ceiling environment. This was the case in three separate sets of measurements made at two ages using two different measurement techniques and two groups of animals. The magnitude of the effect, however, was small and may not have been sufficient to fully compensate for the imposed local hyperopia. To examine this we must calculate the difference in the dioptric challenges offered by the high and low ceilings. For simplicity, consider the challenge $60^{\circ}$ into the upper field, which was the greatest eccentricity

*A problem here is that the chicken's eyes are not normally horizontal but point downwards slightly. In ascessing the impact of the upper and lower visual fields on the depth of the vitreous chamber it was essential to partition the latter with respect to the horizontal rather than with respect to the optic axis. In the primary position. the horizon strikes the retina $9^{-}$below the optic axis (J. C. Letelier, personal communication), and this angle was used to define the boundary between dorsal and ventral retina. at which reliable data could be obtained with our techniques. Geometry indicates that the viewing distance $60^{\circ}$ into the upper field is twice the vertical distance between the eye and the ceiling, so that if the vertical distance were, say, $5 \mathrm{~cm}$ the viewing distance at $60^{\circ}$ would be $10 \mathrm{~cm}$ and hence the dioptric challenge would be $10 \mathrm{D}$. In the high-ceiling environment the ceiling was fixed at $3 \mathrm{~m}$ above the floor, while in the lowceiling enclosure it was variable, being adjusted every few days as the animals grew so as to maintain it approx. $5 \mathrm{~cm}$ above the animals' heads. Thus, the dioptric challenge $60^{\circ}$ into the upper field would be slightly more than $1 / 6 \mathrm{D}$ in the high-ceiling enclosure and range perhaps as high as $10 \mathrm{D}$ in the low-ceiling enclosure.

An obvious complication here is that the dioptric challenge offered by the low ceiling would depend critically on the animals' posture, which was not monitored. We can, however, estimate the likely challenge. If we assume that the refraction error in the lower visual field is fully determined by the height of the animal's eyes above the floor, as suggested for the pigeon by Fitzke et al. (1985) and for several other avian species by Erichsen and Hodos (1989), then, as seen in the Results, we obtain estimates of these heights that average $16 \mathrm{~cm}$ at 8 weeks of age and $24 \mathrm{~cm}$ at 12 weeks. Given that the low ceilings were 30 and $35 \mathrm{~cm}$, respectively, above the floor, this implies that the eyes of the two age groups were $14 \mathrm{~cm}$ and $11 \mathrm{~cm}$, respectively, below this ceiling, suggesting a dioptric challenge $60^{\circ}$ into the upper field of 3.6 and $4.5 \mathrm{D}$, respectively, in the two age groups. Similar reasoning applied to the high ceiling suggests a dioptric challenge $60^{\circ}$ into the upper field in this environment of about $\mathrm{C} .2 \mathrm{D}$ for both age groups. Thus, according to these arguments, at $60^{\circ}$ the challenge offered by the low ceiling exceeded that offered by the high ceiling by 3.4 and 4.3 D, respectively, for the two age groups. At this angle we found that the low-ceiling birds were on average more myopic than the highceiling ones by 1.8 and $2.1 \mathrm{D}$, respectively, in these two groups. The clear suggestion is that the additional myopia observed in the lowceiling birds would compensate for about $50 \%$ of the additional hyperopic challenge offered by this environment.

Of course, if the birds did not compensate completely for the challenge in the lower field it would mean that they had held their heads lower than we have assumed and hence would have compensated more fully in the upper field 
than our estimates suggest. For this reason the relative potency of the growth control mechanism in the dorsal and ventral regions of the retina cannot be firmly established from our data. That the sensitivity of the growth control mechanism to visual experience may not be homogeneous across the retina is suggested by the finding that deprivation of the temporal half of the retina results in a greater growth response than a similar deprivation of the nasal half (Wallman et al., 1987).

The myopia that results from pattern deprivation during development seems to be largely due to an increase in the depth of the vitreous chamber (Wiesel \& Raviola, 1977, 1979; Sommers et al., 1978; O'Leary \& Millodot, 1979; Yinon et al., 1980; Hoyt et al., 1981; Hodos \& Kuenzel, 1984; Hayes et al., 1986; Osol et al., 1986; Lauber \& Oishi, 1987; Troilo et al., 1987; Wallman et al., 1987; PickettSeltner et al., 1987; Gottlieb et al., 1987; PickettSeltner et al., 1988). In the present study, the difference in the dorso-ventral symmetry of the refractions in the low- and high-ceiling birds was associated with an analogous difference in the symmetry of the vitreous chamber, although the demonstration of the latter was complicated by a difference in the overall size of the two groups of birds. The key question now is whether these morphological differences could entirely account for the differences in refractive error. For this, we considered the retina at $30^{\circ}$ (the most eccentric point at which we had confidence in our axial schematic eye), where the measured difference in the depth of the vitreous chamber averaged $0.11 \mathrm{~mm}$ and the measured difference in the refractive error averaged 1.0 D. The axial length here was $14.25 \mathrm{~mm}$, suggesting a second focal length of $12.1 \mathrm{~mm}$ (Wallman \& Adams, 1987). An emmetropic eye with these dimensions would have $110 \mathrm{D}$ of optical power, and adding $0.11 \mathrm{~mm}$ to the depth of the vitreous chamber would result in a myopia of $1.1 \mathrm{D}$, which closely approximates the difference in refractive error actually observed.

However, it is clear that our measures of the depth of the vitreous chamber do not always correlate well with our measures of the refractive error at eccentric locations, and several factors may bear on this. Firstly, these measurements were made to the external surface of the eye and thus ignore possible differences in the thickness of the sclera, choroid and retina. Secondly, current models of the optics of the chicken's eye are only valid close to the optic axis. For example, even the most recently published model predicts much more peripheral myopia than is actually observed (Schaeffel \& Howland, 1988).

We hypothesize that the additional myopia in the upper visual field of the low-ceiling birds is an adaptive response mediated by a local growth control mechanism that adjusts the distance to the retina in different parts of the eye to minimize local refractive error. Thus, we envisage a negative feedback mechanism sensing retinal image quality and working to improve it by promoting, or reducing, local growth of the sclera. As pointed out in the Introduction, this control system can distinguish myopic refractive errors from hyperopic ones, though which of the several possible cues available is actually used remains unclear at present. A complication might arise if the refractive error is excessive: since refractive error reduces image contrast, somewhat like a low-pass spatial frequency filter (Hopkins, 1955), excessive refractive error might, in effect, result in pattern deprivation. This raises the possibility that the myopia in our experiments is a response to deprivation, in which the system is denied any true refractive error signal. In this event it would be appropriate to view the myopia as a pathological response, though it is not clear why the growth control system would interpret the absence of an error signal to mean that the eye is hyperopic (necessitating growth to compensate) rather than myopic (requiring cessation of growth). We doubt that deprivation featured significantly in our experiments since chicks can recover from deprivation myopia or dark-induced hyperopia of up to $20 \mathrm{D}$ (Wallman \& Adams, 1987; Troilo, 1989), even after section of the optic nerve or lesion of the Edinger-Westphal nucleus-procedures that eliminate all control of accommodation (Troilo, 1989). Thus, even with greater refractive errors than those imposed in our experiments, the eye can halt or accelerate its growth appropriately, implying that the degree of ametropia present did not in itself constitute a deprivation that increased eye growth and produced myopia.

Our finding that the refractive error can be altered selectively in a restricted region of the visual field by changing the viewing distance there is consistent with a hypothesis propounded by Wallman et al. (1987). This hypothesis envisioned a local, visually mediated growth control mechanism that normally operates to match the refractive errors across the visual field 
to the customary viewing conditions. Such a mechanism is more easily applied to the lower visual field than to the upper where the viewing distances are generally less well defined. Indeed, it is not clear what images fall on the ventral retina in natural settings and hence are available to guide the normal growth. One might expect that the sky would frequently possess little texture and that exposure to overhanging bushes, trees and the like would be intermittent. Presumably, the eye is able to deal with this uncertainty, perhaps by relying predominantly on periods during which relatively sharp contours are present. One indication of this differential weighting of different types of visual experience is the recent finding that the myopia associated with pattern deprivation can be largely prevented by only $2 \mathrm{hr}$ of normal visual experience per day (Nickla, Panos, FugateWentzek, Gottlieb \& Wallman, 1990).

Acknowledgements - It is a pleasure to thank Yuying Zhu for skilled technical assistance. Dr Karen Pettigrew for carrying out the statistical analyses and Dr Sally Hoskins for her comments on an earlier draft. Supported by NIH research grant EY02727.

\section{REFERENCES}

Dixon, W. J. (1983). BMDP statistical software. University of California Press, Los Angeles.

Erichsen, J. T. (1979). How birds look at objects. Doctoral thesis, Oxford University.

Erichsen. J. T. \& Hodos, W. (1989). Lower-field myopia in birds: An adaptation that keeps the ground in focus. Intestigative Ophthalmology and Visual Science (Suppl.), 30. 31 .

Fitzke, F. W., Hayes, B. P., Hodos, W., Holden, A. L. \& Low, J. C. (1985). Refractive sectors in the visual field of the pigeon eye. Journal of Physiology, 369, 33-44.

Glickstein, M. \& Millodot, M. (1970). Retinoscopy and eye size. Science, New York, 168, 605-606.

Gottlieb, N. D., Fugate-Wentzek, L. A. \& Waliman, J. (1987). Different visual restrictions produce different ametropias and different eye shapes. Investigative $O p h$ thalmology and Visual Science, 28, 1225-1235.

Hayes, B. P., Fitzke, F. W., Hodos, W. \& Holden, A. L. (1986). A morphological analysis of experimental myopia in young chickens. Investigative Ophthalmology and Visual Science, 27, 981-991.

Hodos, W. \& Kuenzel, W. J. (1984). Retinal-image degradation produces ocular enlargement in chicks. Investigative Ophihalmology and Visual Science, 25, 652-659.

Hodos, W., Fitzke, F. W., Hayes, B. P. \& Holden, A. L. (1985). Experimental myopia in chicks: Ocular refraction by electroretinography. Investigative Ophthalmology and Visual Science, 26, 1423-1430.

Hopkins. H. H. (1955). The frequency response of a defocused optical system. Proceedings of the Royal Society of London A.. 23I. 91-103.

Hoyt, C. S., Stone, R. D., Fromer, C. \& Bildon, F. A.
(1981). Monocular axial myopia associated with neonatal eyelid closure in human infants. American Journal of Ophthalmology, 91. 197-200.

Hughes, A. (1979). The artefact of retinoscopy in the rat and rabbit eye has its origin at the retina/vitreous interface rather than in longitudinal chromatic aberration. Vision Research, 19, 1293-1294.

Johnson, C. A., Post, R. B., Chalupa, L. M. \& Lee, T. J. (1982). Monocular deprivation in humans: A study of identical twins. Investigative Ophthalmology and Visual Science, 23, 135-138.

Kirby, A. W.. Sutton, L. \& Weiss, H. (1982). Elongation of cat eyes following neonatal lid suture. Investigative Ophthalmology and Visual Science, 22, 274-277.

Lauber, J. K. \& Oishi, T. (1987). Lid suture myopia in chicks. Investigative Ophthalmology and Visual Science, 28, 1851-1858.

McKanna, J. A. \& Casagrande, V. A. (1978). Reduced lens development in lid-suture myopia. Experimental Eye Research, 26, 715-723.

Nathan, J., Kiely, P. M., Crewther, S. G. \& Crewther, D. P. (1985). Disease-associated image degradation and spherical refractive errors in children. American Journal of Optometry and Physiological Optics, 62, 680-688.

Nickla, D. L., Panos, S. N., Fugate-Wentzek, L. A., Gottlieb, M. D. \& Wallman, J. (1990). What attributes of visual stimulation determine whether chick eyes develop deprivation myopia? Investigative Ophthalmology and Visual Science, (Suppl.), 30 (in press).

O'Leary, D. J. \& Millodot, M. (1979). Eyelid closure causes myopia in humans. Experimentia, 35, 1478-1479.

Osol, G., Schwartz, B. \& Foss, D. C. (1986). The effects of photoperiod and lid suture on eye growth in chickens. Investigative Ophthalmology and Visual Science, 27 , 255-260.

Pickett-Seltner, R. L., Sivak, J. G. \& Pasternak, J. J. (1988). Experimentally induced myopia in chicks: Morphometric and biochemical analysis during the first 14 days after hatching. Vision Research, 28, 323-328.

Pickett-Seltner, R. L., Weerheim, J., Sivak, J. G. \& Pasternak, J. (1987). Experimentally induced myopia does not affect post-hatching development of the chick lens. Vision Research, 27, 1779-1782.

Rabin, J., van Sluyters, R. C. \& Malach, R. (1981). Emmetropization: A vision dependent process. Investigative Ophthaimology and Visual Science, 20, 561-564.

Raviola, E. \& Wiesel, T. N. (1978). Effect of dark-rearing on experimental myopia in monkeys. Imestigative Ophthaimology and Visual Science, 17, 485-488.

Robb, R. M. (1977). Refractive errors associated with hemangiomas of the eyelids and orbit in infancy. American Journal of Ophthalmology, 83, 52-58.

Rose, L., Yinon, U. \& Belkin, M. (1974). Myopia induced in cats deprived of distance vision during development. Vision Research, 14, 1029-1032.

Schaeffel. F. \& Howland, H. C. (1988). Visual optics in normal and ametropic chickens. Clinical Visual Science, 3, 83-98.

Schaeffel, F., Farkas, L. \& Howland, H. C. (1986). Infrared photoretinoscope. Applied Optics, 26, 1505-1509.

Schaeffel, F., Glasser, A. \& Howland, H. C. (1988) Accommodation, refractive error and eye growth in chickens. Vision Research, 28, 639-657.

Sherman, S. M.. Norton, T. T. Casagrande, V. A. (1977). Myopia in the lid-sutured tree shrew (Tupaia glis). Brain Research, 128. 154-157. 
Sivak, J. G. (1976). The accommodative significance of the "ramp" retina of the eye of the stingray. Vision Research, 16. 945-950.

Smith. E. L.. Harwerth, R. S., Crawford, M. L. J. \& von Noorden, G. K. (1987). Observations on the effects of form deprivation on the refractive status of the monkey. Investigative Ophthalmology and Visual Science, 28, 1236-1245.

Sommers, D., Kaiser-Kupfer, M. 1. \& Kupfer, C. (1978). Increased axial length of the eye following neonatal lid suture as measured with A-scan ultrasonography. Investigative Ophthalmology and Visual Science, (ARVO Suppl.). 17, 295.

Troilo, D. (1989). The visual control of eye growth in chicks. Doctoral thesis, City University of New York.

Troilo, D. \& Wallman, J. (1987). Changes in corneal curvature during accommodation in chicks. Vision Research, 27, 241-247.

Troilo, D., Gottlieb, M. D. \& Wallman, J. (1987). Visual deprivation causes myopia in chicks with optic nerve section. Current Eye Research, 6, 993-999.

Wallman, J. \& Adams, J. I. (1987). Developmental aspects of experimental myopia in chicks: Susceptibility, recovery and relation to emmetropization. Visual Research, 27, 1139-1163.

Wallman, J, Adams, J. I. \& Trachtman, J. N. (1981). The eyes of young chickens grow toward emmetropia. Investigative Ophthalmology and Visual Science, 20. $557-561$.

Wallman, J., Turkel, J. \& Trachtman, J. (1978). Extreme myopia produced by modest change in early visual experience. Science, New York, 201, 1249-1251.

Wallman, J., Gottlieb, M. D., Rajaram, V. \& Fugate-
Wentzek, L. (1987). Local retinal regions control local eye growth and myopia. Science, New' York, 237, 1-108.

Walls, G. L. (1942). In The vertebrate eye and its adaptive radiation. Cranbrook Institute of Science, Bloomfield Hills, Mich.

Wiesel, T. N. Raviola. E. (1977). Myopia and eye enlargement after neonatal lid fusion in monkeys. Nature, London, 266, 66-68.

Wiesel, T. N. \& Raviola, E. (1979). Increase in axial length of the macaque monkey eye after corneal opacification. Investigative Ophthalmology and Visual Science, 18. 1232-1236.

Wildsoet, C. F. \& Pettigrew, J. D. (1988). Experimental myopia and anomalous eye growth patterns unaffected by optic nerve section in chickens: Evidence for local control of eye growth. Clinical Vision Sciences 3, 99-107.

Wilson, J. R. \& Sherman, S. M. (1977). Differential effects of early monocular deprivation on binocular and monocular segments of cat striate cortex. Journal of Neurophysiology, 40, 891-903.

Yinon, U., Rose, L. \& Shapiro, A. (1980). Myopia in the eye of developing chicks following monocular and binocular lid closure. Vision Research, 20, 137-141.

Young, F. A. (1961a). The effect of restricted visual space on the primate eye. American Journal of Ophthalmology. $52,799-806$.

Young, F. A. (1961b). The development and retention of myopia by monkeys. American Journal of Optometry and Archives of the American Academy of Optometry, 38. 545-555.

Young, F. A. (1963). The effect of restricted visual space on the refractive error of the young monkey eye. Investigative Ophthalmology and Visual Science, 2. 571-577. 